\title{
Latent Variable Analysis of Causal Interactions in Atrial Fibrillation Electrograms
}

\author{
David Luengo ${ }^{1}$, Víctor Elvira ${ }^{2}$ \\ ${ }^{1}$ Universidad Politécnica de Madrid, Madrid, Spain \\ ${ }^{2}$ Télécom Lille, Univ. Lille, CNRS, CRIStAL, Lille, France
}

\begin{abstract}
Multi-channel intracardiac electrocardiograms of atrial fibrillation $(A F)$ patients are acquired at the electrophysiology laboratory in order to guide radiofrequency $(R F)$ ablation surgery. Unfortunately, the success rate of $R F$ ablation is still moderate, since the mechanisms underlying AF initiation and maintenance are still not precisely known. In this paper, we use an advanced machine learning model, the Gaussian process latent force model (GP$L F M$ ), to infer the relationship between the observed signals and the unknown (latent or exogenous) sources causing them. The resulting GP-LFM provides valuable information about signal generation and propagation inside the heart, and can then be used to perform causal analysis. Results on realistic synthetic signals, generated using the FitzHugh-Nagumo model, are used to showcase the potential of the proposed approach.
\end{abstract}

\section{Introduction}

The term atrial fibrillation (AF) is commonly used by cardiologists to denote a family of cardiac arrhythmias characterized by a rapid and unsynchronized contraction of the atria. In spite of its epidemic nature, and the large number of studies performed over the last decades, the mechanisms underlying AF initiation and maintenance are still not precisely known [1-3]. One of the leading hypotheses (rotor theory) states that specific areas of the myocardium may be responsible for AF initiation and maintenance [4]. Radiofrequency (RF) catheter ablation intends to terminate $\mathrm{AF}$ (and prevent its recurrence) by targeting these arrhythmogenetic areas. However, no consensus has been attained yet on which areas should be ablated, the success rate of a single procedure is still unsatisfactory, and its relative effectiveness w.r.t. the use of antiarrhythmic drugs remains controversial [5-8].

In this paper, we use an advanced machine learning model, the Gaussian process latent force model (GP-LFM) [9-11], to describe the intracardiac electrocardiograms (a.k.a. electrograms (EGMs)) acquired during RF ablation at the electrophisiology laboratory. The GP-LFM assumes that the observed EGMs have been generated by some unknown signals, latent forces (LFs), and tries to learn both those LFs, which are modelled as Gaussian processes (GPs) [12], and the input-output mechanism, which is cast within the linear convolution framework. The resulting GP-LFM provides valuable information about signal generation and propagation inside the heart, and can then be used to perform causal analysis between the observed EGMs and the inferred LFs. Note that Granger causality (G-causality) maps were already built in [13,14], whereas a hierarchical G-causality approach was developed in $[15,16]$. However, none of these approaches takes into account that the observed EGMs have been generated by one or more exogenous sources (i.e., the LFs), as we do here. Results on realistic synthetic signals, generated using the FitzHugh-Nagumo model, are used to showcase the potential of the proposed approach.

\section{Gaussian Process Latent Force Model}

Let us assume that we have a multi-variate time series composed of $Q$ interrelated signals, $y_{q}(t)$ for $q=$ $1, \ldots, Q$, corresponding to EGMs recorded at a single heart site during RF catheter ablation therapy. The GPLFM assumes that the observed output EGMs have been generated by the linear convolution of a set of unknown input latent signals, which are modelled as independent Gaussian processes, with a set of smoothing kernels, which are derived from basic knowledge of the problem. From a mathematical point of view, each of the $Q$ outputs (EGMs) is modelled as

$$
y_{q}(t)=\sum_{r=1}^{R} S_{r, q} y_{r, q}(t)+w_{q}(t) \text {, }
$$

where $S_{r, q}$ represents the coupling strength between the $r$ th LF, $f_{r}(t)$, and the $q$-th output; $w_{q}(t) \sim N\left(0, \eta_{q}^{2}\right)$ is the 


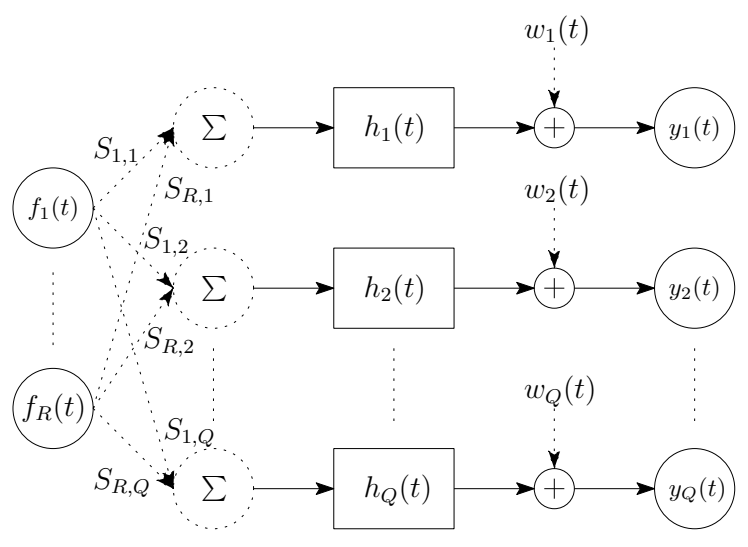

Figure 1. Block diagram of the Gaussian Process Latent Force Model (GP-LFM).

additive white Gaussian noise (AWGN) term; and

$$
y_{r, q}(t)=f_{r}(t) * h_{q}(t)=\int_{0}^{t} f_{r}(\tau) h_{q}(t-\tau) \mathrm{d} \tau
$$

is the $r$-th pseudo-output for the $q$-th EGM (i.e., the part of the $q$-th output that can be explained by the $r$-th LF), with $h_{q}(t)$ denoting the $q$-th smoothing kernel. Fig. 1 provides a graphical representation of the GP-LFM.

In order to complete the statistical description of the model, we must define the latent functions and the smoothing kernels. On the one hand, the $R \operatorname{LFs}\left(f_{r}(t)\right.$ for $r=$ $1, \ldots, R)$ are modelled as independent Gaussian processes (IGPs) with a zero mean function, $\mu_{f_{r}}(t)=0$, and an arbitrary auto-covariance function (ACF), $k_{f_{r} f_{r}}\left(t, t^{\prime}\right)$, which encodes our a priori knowledge about basic characteristics of the LFs such as continuity, smoothness, stationarity, etc. Although several choices for $k_{f_{r} f_{r}}\left(t, t^{\prime}\right)$ are possible (e.g., see [9-11]), in the following we consider only the squared exponential, RBF or Gaussian auto-covariance function:

$$
k_{f_{r} f_{r}}\left(t^{\prime}-t\right) \propto \exp \left(-\frac{\left(t^{\prime}-t\right)^{2}}{\ell_{r}^{2}}\right),
$$

where the hyperparameter $\ell_{r}$ controls the length-scale (i.e., the extent of temporal correlation) of the process. On the other hand, the smoothing kernel encodes our knowledge about the linear system that relates the unknown LFs and the observed EGMs. In this paper, we consider one of the simplest possibilities: the smoothing kernel associated to a first order linear ordinary differential equation with constant coefficients [9],

$$
h_{q}(t)=\exp \left(-D_{q} t\right),
$$

where $D_{q}$ is a parameter which is related to the decay speed of the system. This smoothing kernel acts as a low-pass filter on the input LF, with a potentially different bandwidth (controlled by the decay parameter $D_{q}$ ) for each of the observed outputs.

\section{Granger Causality (G-Causality)}

Granger causality (G-causality) measures the increase in predictability on the future outcome of a signal, given the past values of another signal, w.r.t. the predictability attained by taking into account only past values of itself [17]. We are interested in determining whether each of the LFs is able to provide a statistically significant forecast of each of the outputs. Let us assume that we have $Q$ EGMs that have been acquired by uniformly sampling the underlying continuous signals with a sampling frequency $f_{s}=1 / T_{s}$, $y_{q}[n]=y_{q}\left(n T_{s}\right)$ for $0 \leq n \leq N-1$, and that we have inferred the $R$ LFs at those same instants, $f_{r}[n]=f_{r}\left(n T_{s}\right)$. Then, we can apply the G-causality methodology to determine the increase in predictability on the future outcome of the $q$-th EGM, $y_{q}[n]$ for $1 \leq q \leq Q$, given the past values of the $r$-th latent signal, $f_{r}[n]$ with $1 \leq r \leq R$, w.r.t. the predictability achieved by taking into account only the past values of $y_{q}[n]$. In order to achieve this goal, we first build a linear autoregressive (AR) predictor for $y_{q}[n]$ given its past samples (i.e., the $q$-th self-predictor),

$$
\hat{y}_{q}[n]=\sum_{m=1}^{M_{q q}} \alpha_{q q}[m] y_{q}[n-m],
$$

where $M_{q q}$ is the order of the $q$-th self-predictor and $\alpha_{q q}[m]$ are the coefficients of the model. Similarly, let us define the linear AR predictor for $y_{q}[n]$ given the past samples of both $y_{q}[n]$ and $f_{r}[n]$ (i.e., the cross-predictor from the $r$-th LF to the $q$-th EGM) as

$\hat{y}_{r \rightarrow q}[n]=\sum_{m=1}^{M_{q q}} \alpha_{q q}[m] y_{q}[n-m]+\sum_{m=1}^{M_{r q}} \alpha_{r q}[m] f_{r}[n-m]$,

where $M_{r q}$ is the order of the predictor from the $r$-th LF to the $q$-th EGM, and $\alpha_{r q}[m]$ denotes its coefficients. The residual errors of the two predictors in (5) and (6) are $\varepsilon_{q}[n]=y_{q}[n]-\hat{y}_{q}[n]$ and $\varepsilon_{r \rightarrow q}[n]=y_{q}[n]-\hat{y}_{r \rightarrow q}[n]$ respectively. The G-causality strength is then measured by the logarithm of the ratio of these two variances [18]:

$$
G_{\ell \rightarrow q}=\ln \frac{\operatorname{Var}\left(\varepsilon_{q}[n]\right)}{\operatorname{Var}\left(\varepsilon_{\ell \rightarrow q}[n]\right)} .
$$

Using these pairwise values, we can build a $G$-causality strength matrix, $\mathbf{G}$, whose $(r, q)$-th entry is ${ }^{1}$

$$
\mathbf{G}_{r, q}= \begin{cases}G_{r \rightarrow q}, & r \neq q ; \\ 0, & r=q .\end{cases}
$$

Finally, note that we should add a causality link from $r$ to $q$ only when the decrease in the residual's noise variance

\footnotetext{
${ }^{1}$ Note that $\operatorname{Var}\left(\varepsilon_{q \rightarrow q}[n]\right)=\operatorname{Var}\left(\varepsilon_{q}[n]\right)$, since $\hat{y}_{q \rightarrow q}[n]=\hat{y}_{q}[n]$, and thus $G_{q \rightarrow q}[n]=\ln 1=0$ and the definition in (8) is consistent with (7).
} 


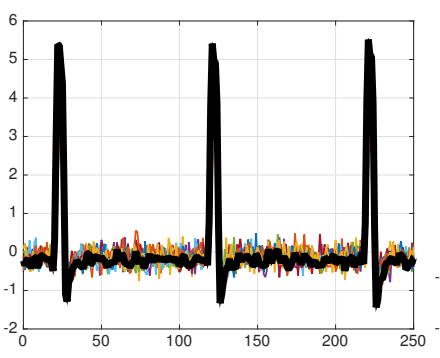

(a)

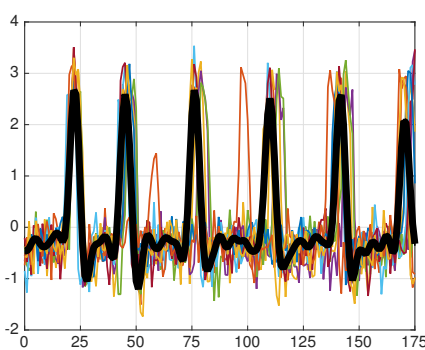

(b)

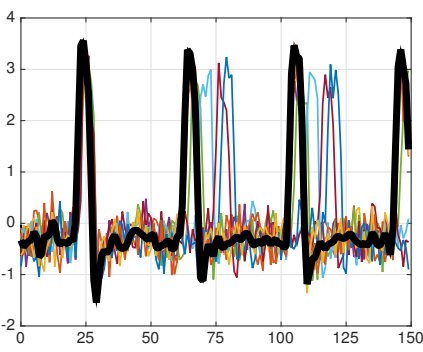

(c)

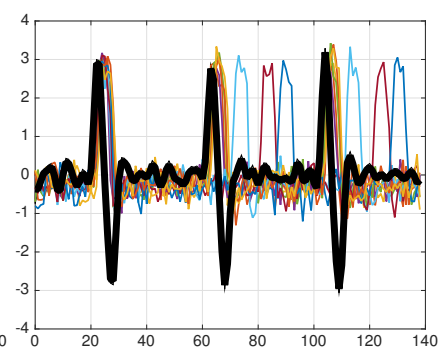

(d)

Figure 2. Example of the aligned signals for the four cases considered (colored thin lines) and the LF learnt (black thick line). (a) Flat. (b) Circular. (c) Rotor 1. (d) Rotor 2.

from (5) to (6) is statistically significant. In order to construct this causality graph, we may define the $G$-causality connection matrix, $\mathbf{C}$, whose $(r, q)$-th element is

$$
C_{r \rightarrow q}=\chi_{p}\left(G_{r \rightarrow q}\right),
$$

where $\chi_{p}(\cdot)$ is a threshold function that determines, when applied to $G_{r \rightarrow q}$ and for a pre-defined $p$-value, whether the $r$-th LF is statistically signiticant in predicting the $q$-th output (i.e., $C_{r \rightarrow q}=1$ ) or not (i.e., $C_{r \rightarrow q}=0$ ).

\section{Numerical Simulations}

In this section, we use several realistic synthetic signals to showcase the potential of the proposed approach. These signals have been generated using a grid of interconnected elements that emulate the behaviour of heart tissue using the FitzHugh-Nagumo model [19], and placing a virtual lasso cathether with $Q=9$ monopolar sensors at some point within that grid. We have simulated four signals:

- Flat, which corresponds to the flat propagation wavefront typically obtained when we have a regular rhythm and the catheter is placed far away from the source.

- Circular, which corresponds to the circular propagation wavefront typically obtained when we have a regular rhythm and the catheter is placed close to the source.

- Rotor 1 and Rotor2, which corresponds to the spiral propagation wavefront obtained when a rotor (turning anticlockwise and clockwise, respectively) is anchored within the area enclosed by the catheter.

In all cases, the clean signals are contaminated by AWGN with an SNR $=10 \mathrm{~dB}$. The Multi-output Gaussian Processes - MATLAB Software ${ }^{2}$ has been used to construct the model for the signals, assuming a single LF (i.e., $R=1$ ). Since the kernel used does not consider a different delay for each of the outputs, a simple algorithm (based on the detection of the first QRS complex by means of a threshold) is used to estimate their relative delay and align all the signals. A stochastic gradient descent method

\footnotetext{
${ }^{2}$ https://sheffieldml.github.io/multigp/
}

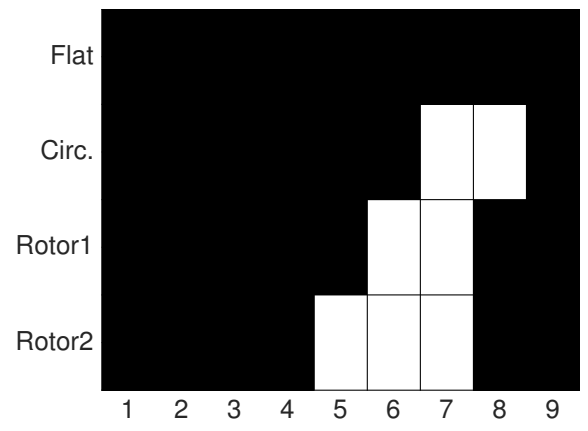

Figure 3. Causal connectivity among the inferred LF, $f(t)$, and each of the $Q=9$ EGMs for the four cases considered (flat, circular, rotor 1 and rotor 2). Note that a black square implies causality (i.e., $C_{r \rightarrow q}=1$ ), whereas a white square indicates the lack of causality (i.e., $C_{r \rightarrow q}=0$ ).

is then applied to learn the hyperparameters $\left(\ell_{r}, S_{r q}, D_{q}\right.$ and $\sigma_{q}$ for $r=1$ and $q=1, \ldots, Q=9$ ), using all the samples from the available EGMs $\left(N_{q}\right.$ for $\left.q=1, \ldots, Q=9\right)$ as the training set. After this training stage, the single LF, $f(t)=f_{1}(t)$, can be easily recovered using standard GP formulas [12]. Finally, the Granger causal connectivity toolbox (see [20]) is used to obtain the desired G-causality relationships with $M_{q q}=M_{r q}=10$ and $p=10^{-4} .3$

Fig. 2 shows the $Q=9$ aligned signals for the four cases considered (colored thin lines) and the LF learnt (black thick line). On the one hand, note the perfect alignment among the single LF and all the EGMs attained for the flat propagation case, which implies that the model has been able to capture all the relevant information in the outputs. On the other hand, note that some activations in the EGMs do not correspond to peaks in the LF for the remaining three cases, implying that the model has missed some relevant features of the outputs. Fig. 3 confirms this fact by showing the causal connectivity among the inferred LF, $f(t)$, and each of the $Q=9$ EGMs for the four cases con-

\footnotetext{
${ }^{3}$ Several values for these parameters were tested, but the performance seemed to be largely insensitive to the values of $M_{q q}=M_{r q}$ (as long as large enough values were used) and $p$.
} 
sidered. Note that a causal link among the LF and all the EGMs is found for the first case (flat propagation), whereas some signals cannot be fully explained by using $f(t)$ alone in the remaining three cases.

\section{Conclusions and Future Lines}

In this paper, we have applied an advanced machine learning model, the Gaussian process latent force model (GP-LFM), to describe the intracardiac electrocardiograms acquired during RF ablation surgery. The GP-LFM allows us to recover the unknown latent forces (LFs) that have generated the observed outputs, as well as the inputoutput interaction mechanism, and perform causal analysis. Promising results have been attained on synthetic signals, generated using the FitzHugh-Nagumo model. Future lines include extending the GP-LFM (e.g., adding a variable delay for the different outputs and developing novel input-output kernels), and testing it on real signals.

\section{Acknowledgements}

This work has been supported by the Spanish government through the OTOSiS project (TEC2013-41718$\mathrm{R})$, and the BBVA Foundation's "I Convocatoria de Ayudas Fundación BBVA a Investigadores, Innovadores y Creadores Culturales". The authors would also like to thank Gonzalo Ríos-Muñoz for providing the synthetic signals that were used in this study.

Address for correspondence:

David Luengo

Universidad Politécnica de Madrid, ETSIS de Telecomunicación, Desp. 7009, Ctra. de Valencia, Km. 7, 28031 Madrid (Spain)

E-Mail: luengod@ieee.org

\section{References}

[1] Nattel S, Li D, Yue L. Basic mechanisms of atrial fibrillation-very new insights into very old ideas. Annual Review of Physiology January 2000;62(1):51-77.

[2] Nattel S, Shiroshita-Takeshita A, Brundel BJ, Rivard L. Mechanisms of atrial fibrillation: Lessons from animal models. Progress in Cardiovascular Diseases July/August 2005;48(1):9-28.

[3] Krummen DE, Narayan SM. Mechanisms for the initiation of human atrial fibrillation. Heart Rhythm August 2009; 6(8, Supplement):S12-S16.

[4] Jalife J, Berenfeld O, Mansour M. Mother rotors and fibrillatory conduction: a mechanism of atrial fibrillation. Cardiovascular research 2002;54(2):204-216.

[5] Cosedis Nielsen J, Johannessen A, Raatikainen P, Hindricks G, Walfridsson H, Kongstad O, Pehrson S, Englund A, Hartikainen J, Mortensen LS, et al. Radiofrequency ablation as initial therapy in paroxysmal atrial fibrillation. New England Journal of Medicine 2012;367(17):1587-1595.

[6] Ganesan AN, Shipp NJ, Brooks AG, Kuklik P, Lau DH, Lim HS, Sullivan T, Roberts-Thomson KC, Sanders P. Longterm outcomes of catheter ablation of atrial fibrillation: a systematic review and meta-analysis. Journal of the American Heart Association 2013;2(2):e004549.

[7] Jones DG, Haldar SK, Hussain W, Sharma R, Francis DP, Rahman-Haley SL, McDonagh TA, Underwood SR, Markides V, Wong T. A randomized trial to assess catheter ablation versus rate control in the management of persistent atrial fibrillation in heart failure. Journal of the American College of Cardiology 2013;61(18):1894-1903.

[8] Verma A, Jiang Cy, Betts TR, Chen J, Deisenhofer I, Mantovan R, Macle L, Morillo CA, Haverkamp W, Weerasooriya R, et al. Approaches to catheter ablation for persistent atrial fibrillation. New England Journal of Medicine 2015;372(19):1812-1822.

[9] Alvarez MA, Luengo D, Lawrence ND. Latent force models. In International Conference on Artificial Intelligence and Statistics. 2009; 9-16.

[10] Alvarez MA, Luengo D, Titsias MK, Lawrence ND. Efficient multioutput gaussian processes through variational inducing kernels. In International Conference on Artificial Intelligence and Statistics. 2010; 25-32.

[11] Álvarez MA, Luengo D, Lawrence ND. Linear latent force models using gaussian processes. Pattern Analysis and Machine Intelligence IEEE Transactions on 2013; 35(11):2693-2705.

[12] Rasmussen CE, Williams CKI. Gaussian Processes for Machine Learning. New York: The MIT Press, 2006.

[13] Rodrigo M, Liberos A, Guillem M, Millet J, Climent AM. Causality relation map: a novel methodology for the identification of hierarchical fibrillatory processes. In Computing in Cardiology, 2011. IEEE, 2011; 173-176.

[14] Rodrigo M, Guillem MS, Liberos A, Millet J, Berenfeld O, Climent AM. Identification of fibrillatory sources by measuring causal relationships. In Computing in Cardiology (CinC), 2012. IEEE, 2012; 705-708.

[15] Luengo D, Gonzalo R, et al. Causality analysis of atrial fibrillation electrograms. In 2015 Computing in Cardiology Conference (CinC). IEEE, 2015; 585-588.

[16] Luengo D, Gonzalo R, Art A, et al. A hierarchical algorithm for causality discovery among atrial fibrillation electrograms. In 2016 IEEE International Conference on Acoustics, Speech and Signal Processing (ICASSP). IEEE, 2016; 774-778.

[17] Granger CWJ. Investigating causal relations by econometric models and cross-spectral methods. Econometrica 1969; 37:424-438.

[18] Geweke J. Measures of conditional linear dependence and feedback between time series. Journal of the American Statistical Association 1984;79:907-915.

[19] Keener JP, Sneyd J. Mathematical physiology, volume 1. Springer, 1998.

[20] Seth AK. A matlab toolbox for granger causal connectivity analysis. Journal of neuroscience methods 2010; 186(2):262-273. 